\title{
Fear of COVID-19 and work-quality of life among nurses: The mediating role of psychological well-being
}

\author{
Ahmet Maslakçı ${ }^{a^{*}}$, Lütfi Sürücü̈ ${ }^{b}$ and Harun Sesen ${ }^{c}$
}

${ }^{a}$ Cyprus Science University, Turkey

${ }^{b}$ European Leadership University, Turkey

${ }^{c}$ European University of Lefke, Turkey

\section{H R O N I C L E}

Article history:

Received: December 20, 2020

Received in revised format:

January 202021

Accepted: March 22, 2021

Available online:

March 22, 2021

Keywords:

COVID-19

Psychological well-being

Quality of work life

Nursing

\section{A B S T R A C T}

This study was conducted in order to analyze the effect of the nurses' quality of work life based on fear about COVID-19 and examine the psychological well-being as a moderating variable in this relationship. The survey questionnaire was administered among nurses between 1 November 2020 and 14 November 2020. The self-report survey comprised the nurse information survey, Fear of COVID-19 Scale, work quality of life scale, and psychological well-being scale as data collection tools. Data were obtained from 339 nurses. The findings show that fear of COVID-19 negatively affects nurses' quality of work life. It has been determined that PWB plays a moderating role in this relationship. While the fear of COVID-19 negatively affects the quality of work life in nurses with low psychological well-being, there is not any kind of significant effect on the quality of work life in nurses with high psychological well-being. This result shows that as the psychological wellbeing of nurses' increases, fear of COVID-19 effect on quality of work life decreases. The results of the study show that responses designed to enhance psychological well-being can enhance nurses' working conditions that could reduce the negative effects of the fear of COVID-19. There is an urgent need for clinical and policy strategies to help increase nurses' PWB in order to increase the quality of work life by reducing fear and also anxiety among nurses fighting on the front line during COVID-19.

\section{Introduction}

“Coronavirus disease 2019 (COVID-19)", which showed up first in Wuhan, China in 2019 December was announced as a pandemic by the World Health Organization (WHO). It is a public health problem that has affected individuals worldwide. In addition to the economic and social problems it has caused, COVID-19 has had a dangerous effect upon the psychological state of the population (Huang \& Zhao, 2020) and has also created unprecedented challenges that have caused a significant impact on health systems and health practitioners ( $\mathrm{Li}, \mathrm{Zhou}, \& \mathrm{Xu}, 2020)$. In order to restrict the spread of the COVID-19 epidemic, some countries have offered compulsory curfew or social distance. While preventive measures can be effective in preventing the spread of the disease (Swami, Horne, \& Furnham, 2020), the effect of social distance and curfew that include some alterations in daily actions, may have negative effects upon the mental health of the population (Galea, Merchant, \& Lurie, 2020). Nurses have always been at the forefront of healthcare and are now faced with the enormous challenges posed by the COVID-19 epidemic (Li et al., 2020). Nurses tend to experience more stress than other healthcare workers due to heavy workloads, shift work rotation, suffering of patients, and numerous other occupational stressors that play a crucial factor in public health protection, restrain and control the infection and isolation (Smith, Ng, \& Ho Cheung Li, 2020). The mortality rate of COVID-19 and high risk of infection might increase nurses' anxiety and fear, leading to high levels of stress (Labrague \& de los Santos, 2020; Li et al., 2020; Pang, Dan, Jung, Bae, \& Kim, 2020). During the COVID-19 epidemic, it may be crucial

* Corresponding author.

E-mail address: lutfi.surucu@elu.edu.tr (L. Sürücü) 
for nurses to care for subjective psychological well-being in addition to paying attention to their patients (Mo et al., 2020; Tran et al., 2019). Research nurses' health status and their performance have positive relationships (Fronteira \& Ferrinho, 2011; Oyama \& Fukahori, 2015). Additionally, fear, worry, and stress also influence the quality of work life, which attribute to mental satisfaction and well-being (Jin \& Lee, 2020). The quality of work life is a crucial determinant of an individual's working life and quality of life, and it significantly affects work success (Jin \& Lee, 2020). Quality of work life, which is a complex and multidimensional framework (Hsu \& Kernohan, 2006), reflect nurses' attitudes and feelings towards their jobs (Dehghan Nayeri, Salehi, \& Ali Asadi Noghabi, 2011). Depressive symptoms such as stress, anxiety, and worry that affect the quality of work life reduce nurses' concentration and cognitive performance, which may affect their job performance and ultimately lead to a decline in the quality of patient care (Maharaj, Lees, \& Lal, 2019).

Additionally, anxiety and worry caused by the fear of COVID-19 (Ahorsu et al., 2020) can affect nurses' overall well-being (Li et al., 2020), which exposes them to emotional changes related to psychological well-being (Lee, Tzeng, \& Chiang, 2019). Consequently, a decline in psychological well-being (PWB) can give rise to mental illness and emotional fatigue (Divinakumar, Pookala, \& Das, 2014). Literature on the topic indicates that the quality of work environment and mental health of nurses is positively associated with PWB and safety (Lee et al., 2019). Despite the possible impact of PWB on nurses' quality of work has been stated in previous studies, a limited number of researches have empirically examined this hypothesis (Lee, Kang, Cho, Kim, \& Park, 2018). In addition, the effects of PWB on nurses' work environment has not been examined as part of the fear of COVID-19 (Lee et al., 2019). Although they showed advantages of PWB for another group of people, to our knowledge, no researchers have investigated the link between PWB, the fear of COVID-19, and quality of work life in nurses. In addition, there are restricted figures showing the effect of the fear of COVID-19 on nurses' working lives (Jin \& Lee, 2020). Therefore, the overall research of this research was to analyse the factors associated with the quality of work life and the mental well-being of frontline nurses during the COVID-19 outbreak. The main purpose of this research is to contribute to the research targeted at addressing the effects of the fear of COVID-19 on the psychological health of nurses, as stated by Mo et al. (2020). The main outcomes of this research will be of significance to nursing managers, who can provide suggestions to policymakers, and give practical advice on effectively supporting the psychological health of nurses who act at the frontline and maintaining a well-connected nursing labour force, especially throughout the epidemic. Accordingly, the conceptual framework of the research is based on exploring the role of PWB in the link between the fear of COVID-19 and the quality of work life by examining the direct and indirect effects (Fig. 1).

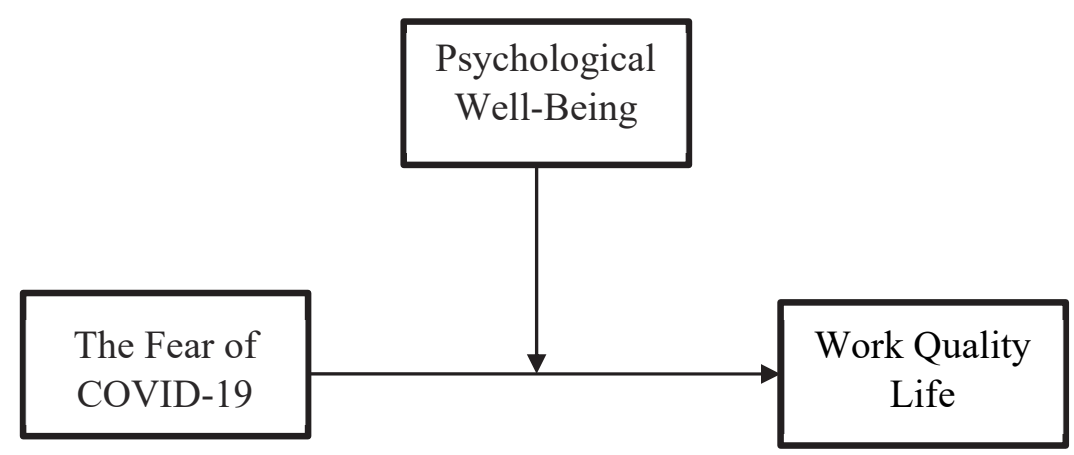

Fig. 1. Conceptual Framework

$\mathrm{H}_{1}$ : The fear of COVID-19 affects work quality life significantly and negatively in nurses.

$\mathrm{H}_{2}$ : The fear of COVID-19 affects psychological well-being significantly and negatively in nurses.

$\mathrm{H}_{3}$ : Psychological well-being affects work quality life significantly and positively in nurses.

$\mathrm{H}_{4}$ : Psychological well-being has a moderating role in the effect of COVID-19 fear in nurses on work quality life.

\section{Methodology}

\subsection{Design and Sample}

The research employed a cross sectional descriptive research design. Data were gained from a state hospital in Nicosia, North of Cyprus over a period of one week in November 2020. The hospital where the research was conducted is the largest hospital in the region with a bed capacity of 500. The sample selection method was not used in the study, and it was aimed to apply a questionnaire to all nurses. Inclusion criteria are nurses participating in the hospital and volunteering to be involved in the survey. Data were obtained from 351 nurses using a self-report survey. The data obtained were evaluated in line with the criteria proposed by Byrne (2016), and 12 questionnaires that were filled incompletely and incorrectly were excluded from the research. A total of 339 questionnaires were analysed.

\subsection{Measurements}

The survey had a total of 18 items, including the nurse information survey, "Fear of COVID-19 Scale", "work quality-of-life scale", and "psychological well-being scale". 
The Nurse Information Questionnaire. This questionnaire comprises four items for determining nurses' age, gender, marital status, and the departments in which they work.

Fear of COVID-19 scale: This scale emerged by Ahorsu et al. (2020), was used in order to determine the level of fear related to COVID-19 in nurses. This scale has been used in several recent studies. It contains seven items on a Likert scale that include 5 points. Items are rated from "1 (Strongly Disagree) to 5 (Strongly Agree)". The Cronbach's alpha coefficient for the scale was .927 .

Work quality-of-life scale: This scale developed by Sirgy (2001), was used to determine the quality of work life within nurses. It contains four items scored on a five-point Likert scale. Items are rated from "1 (Strongly Disagree) to 5 (Strongly Agree)". The Cronbach's alpha coefficient for the scale was .859 .

Psychological well-being scale: This scale developed by Baker and Kim (2020), was used to assess the psychological wellbeing of nurses. The scale contains three items scored on a seven-point Likert scale. Items are rated from "1 (strongly disagree) to 7 (strongly agree)". The Cronbach's alpha coefficient for the scale was .883.

\subsection{Ethical Considerations}

The research was conducted according to standard ethical codes of conduct. The research was confirmed by the Ethics Committee of [blinded for review]. Permission was obtained from the director of the hospital from where the participants were recruited. Participants were told about the background of the research and that their personal information would remain confidential. In order to avoid bias in analysing the responses of the participants, the questionnaires were distributed and collected in closed envelopes.

\subsection{Data Analyses}

"SPSS (Statistical Package for the Social Sciences) statistical software" version 23.0 and "AMOS (Analysis of Moment Structures) statistical software" version 18.0 were used for data analyses. The participants answered all of the items in the survey, and there were no missing data. Means, percentages, standard deviations, and frequencies were used to present descriptive statistical information. The distribution of data was determined by the skewness and kurtosis values. The link between variables was determined using Pearson's correlation coefficient. The fit indices of the proposed model in the study were determined using AMOS statistics software. Process Macro, developed by Hayes (2017) as an extension to SPSS, was used to test the hypotheses. In this study, the significance level was set at $p<.05$.

\section{Results}

\subsection{Participants' Characteristics}

The study sample consisted of 339 nurses working in a state hospital. Among the participants, $296(87.3 \%)$ of the nurses were female and $43(12.7 \%)$ were male; 211 participants were married (62.2\%) and $128(37.8)$ participants were single. Regarding age, $79(23.3 \%)$ participants were 25 years of age or younger, $111(32.7 \%)$ participants' ages ranged between 26-30 years, 98 (28.9\%) participants' ages ranged between 31-40 years, and 51 (15\%) participants were aged 41 years and above. Regarding departments of work, 55 (16.2\%) participants worked in the emergency service, 140 (41.3\%) participants worked in the general ward, 47 (13.9\%) participants worked in the operating room, and $97(28.6 \%)$ participants worked in other departments. The highest mean score of the nurses was on the psychological well-being scale $(4.54 \pm 1.553)$. The average values for the other scales were: work quality-of-life scale ( $3.54 \pm .874)$ and Fear of COVID19 Scale $(3.16 \pm 1.073)$. The results show that the average scores of the scales did not fall below 3 , which is the score associated with indecision of the participants (Table 1).

\subsection{Preliminary Analysis}

Confirmatory factor analysis (CFA) was used the AMOS 18 program to verify the factor structure of the scales included in the study. The CFA results using the maximum possibility method showed that the model had good fit indices (CMIN / $d f=$ $2.503, \mathrm{GFI}=.927, \mathrm{NFI}=.951, \mathrm{IFI}=.970, \mathrm{TFI}=.961, \mathrm{CFI}=.970, \mathrm{RMSEA}=.067)$.

\subsection{Primary Analysis}

Pearson's correlation analysis was run to determine the relationship between the variables in the research.

Table 1

$\underline{\text { Pearson's r Correlations between the Fear of COVID-19, Work Quality-of-Life and Psychological Well-Being ( } \mathrm{n}=339)}$

\begin{tabular}{|c|c|c|c|c|c|}
\hline Variables & Mean & SD & 1. & 2. & 3. \\
\hline 1. Fear of COVID-19 & 3.16 & 1.553 & 1 & & \\
\hline 2. Work quality-of-life & 3.54 & .874 & $-.176^{* *}$ & 1 & \\
\hline 3. Psychological well-being & 4.54 & 1.073 & $-.121 *$ & $.779 * *$ & 1 \\
\hline
\end{tabular}


The demonstrated that the fear of COVID-19 was negatively correlated with work quality of life $(\mathrm{QOL})(r=-.176, p<.001)$ and psychological well-being $(r=-.121, p<.05)$. In addition, the correlation between work QOL and psychological wellbeing was positive $(r=.779, p<.05)$.

\subsection{Regression Analysis}

Process Macro (Model 1), developed by Hayes (2017), was used in order to examine the hypotheses suggested in the study. In the bootstrap regression analysis conducted with Process Macro, the $95 \%$ confidence intervals (5000 bootstrapping option) were chosen.

Table 2

Bootstrap Regression Results

\begin{tabular}{|c|c|c|c|c|c|c|}
\hline Variables & Coeff & $\mathrm{SE}$ & $t$ & $p$ & LLCI & ULCI \\
\hline Constant & 3.5463 & .0295 & 120.032 & .0000 & 3.4881 & 3.6044 \\
\hline Fear of COVID-19 & -.0765 & .0278 & -2.750 & .0063 & -.1312 & -.0218 \\
\hline Psychological well-being & .4275 & .0192 & 22.282 & .0000 & .3898 & .4652 \\
\hline Int_term (TFOC x PWB) & .0431 & .0166 & 2.595 & .0099 & .0104 & .0758 \\
\hline $\mathrm{R}^{2}$ & .6212 & & & & & \\
\hline $\mathrm{R}^{2}$-chng & .0076 & & & & & \\
\hline $\mathrm{F}$ & 6.7330 & & & & & \\
\hline
\end{tabular}

The bootstrap results indicate that the fear of COVID-19 negatively affected work QOL $(\beta=-.0765,95 \%$ CI $=[-.1312,-$ $.0218], t=-2.750, p<.05)$, and psychological well-being played a moderating role in this relationship $(\beta=.0431,95 \% \mathrm{CI}=$ $[.0104,-0758], \mathrm{t}=2.595, p<.05)$. In addition to these findings, the results of the analysis shows that psychological wellbeing affected work quality-of-life significantly and positively $(\beta=.4275,95 \% \mathrm{CI}=[.3898, .4652], t=22.282, p<.05)$. In the light of the findings, H1, H2, H3 and H4 were supported. The simple slope regression graph proposed by Aiken, West, \& Reno (1991) was created in order to extend the analysis results further and to visually observe the effect of the fear of COVID19 on nurses' work QOL and psychological well-being (Fig. 2).

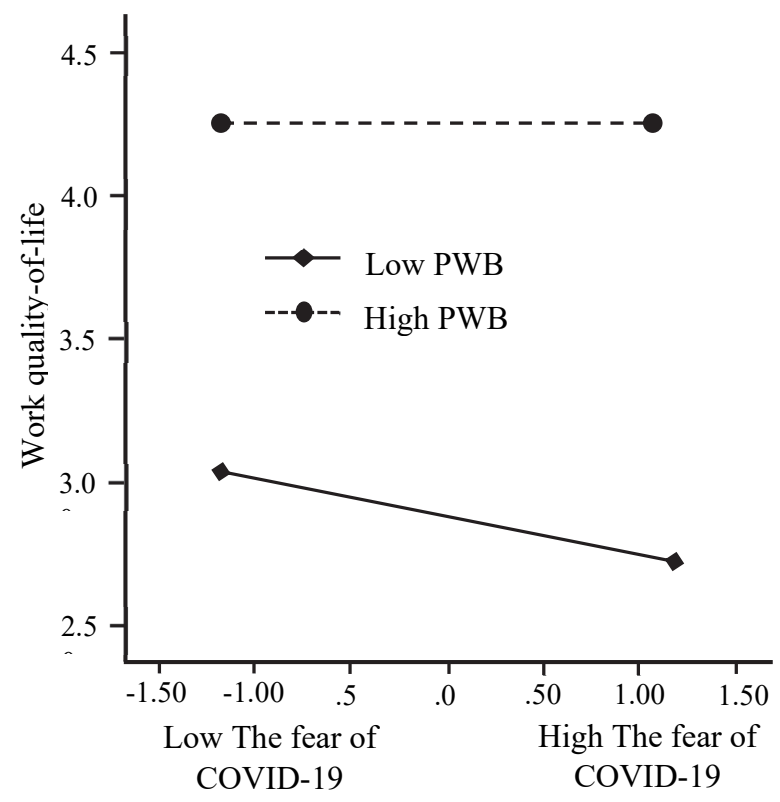

Fig. 2. Interaction effect between psychological well-being and fear of COVID-19 on work quality-of-life

The simple slope regression graph shows that the effect of the fear of COVID-19 on work QOL varied according to the psychological well-being of nurses. Accordingly, in nurses with low psychological well-being, the fear of COVID-19 negatively affected their work QOL $(\beta=-.1427,95 \% \mathrm{CI}=[-.2213,-.0640], t=-3.5688, p=.0004)$, while the fear of COVID-19 had no effect on work QOL in nurses with high psychological well-being $(\beta=-.0048,95 \% \mathrm{CI}=[-.0770, .0675], t=-.1294, p$ $=.8971)$. These results clearly show that as the psychological well-being of nurses increased, the effect of the fear of COVID19 on work QOL decreased.

\section{Discussion}

The outcomes of the research show that the fear of COVID-19 and PWB play a significant role in evaluating nurses' perceptions of the quality of their working environment. The findings revealed that the fear of COVID-19 and PWB scores predicted the quality of work life significantly. 
COVID-19 is a pandemic that continues to affect many people around the world, and as such, frontline nurses are facing stress and anxiety (Li et al., 2020). However, there is a dearth of information about how the fear of COVID-19 affects nurses (Ahorsu et al., 2020; Li et al., 2020). This research can be counted as the first to determine how fear of COVID-19 might affect nurses' work QOL and the moderating role of PWB in this relationship. The results show that the fear of COVID-19 negatively affected nurses' work quality, which is similar to the findings in previous literature (Pang et al., 2020). In a research carried out within the COVID-19 pandemic, Huang and Zhao (2020) determined that healthcare employees experienced high levels of stress compared to the whole population. It has been found that healthcare workers (involving nurses, doctors, and auxiliary workers) experienced fears and concerns during previous epidemics as well (e.g., SARS and MERS virus outbreak) (Lee et al., 2018). In a study conducted by Chua et al. (2004), within the SARS epidemic period, $89 \%$ of healthcare workers reported an increase in their psychological symptoms and anxiety levels in high-risk situations (Chua et al., 2004).

The outcomes of the present research demonstrated that the COVID-19 epidemic is leading to increasing uncertainty and stress throughout high-risk nurses, similar to other pandemics. Pandemics contribute to the increase in nurses' fear and anxiety (Aksoy \& Koçak, 2020) and have negative effects on their quality of work life (Maharaj et al., 2019).

Nurses' psychological health have a crucial role in enhancing safety of their patients and care quality towards them (Lee et al., 2018). In some research, it was demonstrated that the quality of the work conditions have a positive relationship with safety of patients and the patients care quality (Kirwan, Matthews, \& Scott, 2013; Lee et al., 2018). A decrease in working conditions' quality may cause sleep disorders and increase physical and psychological problems among nurses (Wu et al., 2014). Our results indicate that PWB functions as a moderator between the fear of COVID-19 and the quality of the work environment among nurses. According to this, while the fear of COVID-19 negatively affects work QOL in nurses with low psychological well-being, it has no effect on work QOL in nurses with high psychological well-being. These results clearly show that as the psychological well-being of nurses increases, the effect of the fear of COVID-19 on work QOL decreases.

\subsection{Practical Implications}

Nurse managers should work towards improving nurses' working environments and aim to provide them with quality and safe working conditions. Forthcoming interferences at the institutional and national levels are required to enhance mental wellbeing throughout nurses working on the front line of healthcare during COVID-19. In addition, health policy makers need to identify the factors that affect nurses' PWB and provide strategies to help address them. Training policies for nurses should be developed in this area. During the pandemic, a secure and fast information network, readiness and appropriate education, and easy access to protective equipment can help increase nurses' PWB and reduce the negative mental effects caused by the pandemic.

\subsection{Conclusion}

During critical times such as COVID-19, it is necessary to facilitate an increase in the PWB of nurses to enhance the work conditions' quality. In order to decrease the negative effects of nurses' anxiety caused by COVID-19 on their work environment, policies should be developed to help increase their PWB. Further research could explore ways to increase the PWB of nurses during the pandemic. An increase in PWB will help to reduce the anxiety and fear experienced by nurses during the pandemic which would, in turn, improve the quality of care received by patients. Outcomes have significant inferences for nurse education policies aimed at increasing nurses' PWB. Such interventions can lead a to much better nursing work conditions, increased safety of patients.

\subsection{Limitations}

Certain limitations should be considered regarding the present study. First of all this research is only done with nurses at a local hospital in Nicosia, and the findings cannot be generalised to all Cypriot nurses. Second, the data are derived using selfreport tools; therefore, the outcomes linked with the limitations associated with the research of methodology. Finally, some other factors associated with COVID-19, such as social isolation and working hours, may be related to the psychological wellbeing of nurses; however, these factors were not included in the survey due to time constraints.

\section{References}

Ahorsu, D. K., Lin, C. Y., Imani, V., Saffari, M., Griffiths, M. D., \& Pakpour, A. H. (2020). Fear of COVID-19 scale: Development and initial validation. International Journal of Mental Health and Addiction, 1-9. https://doi.org/10.1007/s11469020-00270-8.

Aiken, L. S., West, S. G., \& Reno, R. R. (1991). Multiple regression: Testing and interpreting interactions. Sage.

Aksoy, Y. E., \& Koçak, V. (2020). Psychological effects of nurses and midwives due to COVID-19 outbreak: The case of Turkey. Archives of Psychiatric Nursing, 34(5), 427-433. https://doi.org/10.1016/j.apnu.2020.07.011

Baker, M. A., \& Kim, K. (2020). Dealing with customer incivility: The effects of managerial support on employee psychological well-being and quality-of-life. International Journal of Hospitality Management, 87, 102503. https://doi.org/10.1016/j.ijhm.2020.102503 
Byrne, B. M. (2016). Structural equation modelling with AMOS: Basic concepts, applications, and programming, (3rd ed.). New York: Routledge.

Chua, S. E., Cheung, V., Cheung, C., McAlonan, G. M., Wong, J. W., Cheung, E. P., ... \& Tsang, K. W. (2004). Psychological effects of the SARS outbreak in Hong Kong on high-risk health care workers. The Canadian Journal of Psychiatry, 49(6), 391-393.

Dehghan Nayeri, N., Salehi, T., \& Ali Asadi Noghabi, A. (2011). Quality of work life and productivity among Iranian nurses. Contemporary Nurse, 39(1), 106-118. https://doi.org/10.5172/conu.2011.39.1.106

Divinakumar, K. J., Pookala, S. B., \& Das, R. C. (2014). Perceived stress, psychological well-being and burnout among female nurses working in government hospitals. International Journal of Research in Medical Sciences, 2, 1511-1515. https://doi.org/10.5455/2320-6012.ijrms20141150

Fronteira, I., \& Ferrinho, P. (2011). Do nurses have a different physical health profile? A systematic review of experimental and observational studies on nurses' physical health. Journal of Clinical Nursing, 20, 2404-2424. doi:10.1111/j.13652702.2011.03721.x

Galea, S., Merchant, R. M., \& Lurie, N. (2020). The mental health consequences of COVID-19 and physical distancing: The need for prevention and early intervention. Jama International Medicine, 180(6), 817-818. https://doi.org/10.1001/jamainternmed.2020.

Hayes, A. F. (2017) Introduction to mediation, moderation, and conditional process analysis: A regression-based approach (1562). New York: Guilford Press.

Hsu, M. Y., \& Kernohan, G. (2006). Dimensions of hospital nurses' quality of working life. Journal of Advanced Nursing, 54, 120-131. https://doi.org/10.1111/j.1365-2648.2006.03788.x

Huang, Y., \& Zhao, N. (2020). Generalized anxiety disorder, depressive symptoms and sleep quality during COVID-19 outbreak in China: A web-based cross-sectional survey. Psychiatry Research, 288. doi: 10.1016/j.psychres.2020.112954

Jin, J., \& Lee, E. (2020). Effect of workplace spirituality on quality of work life of nurse cancer survivors in South Korea. Asia-Pacific Journal of Oncology Nursing, 7(4), 346. doi:10.4103/apjon.apjon_36_20

Kirwan, M., Matthews, A., \& Scott, P. A. (2013). The impact of the work environment of nurses on patient safety outcomes: A multi-level modelling approach. International Journal of Nursing Studies, 50(2), $253-263$. https://doi.org/10.1016/j.ijnurstu.2012.08.020

Labrague, L. J., \& de los Santos, J. (2020). Fear of COVID-19, psychological distress, work satisfaction and turnover intention among frontline nurses. Journal of Nursing Management. https://doi.org/10.1111/jonm.13168

Lee, S. M., Kang, W. S., Cho, A. R., Kim, T., \& Park, J. K. (2018). Psychological impact of the 2015 MERS outbreak on hospital workers and quarantined hemodialysis patients. Comprehensive Psychiatry, 87, $123-127$. https://doi.org/10.1016/j.comppsych.2018.10.003

Lee, T. H., Tzeng, W. C., \& Chiang, H. H. (2019). Impact of coping strategies on nurses' well-being and practice. Journal of Nursing Scholarship, 51(2), 195-204. doi:10.1111/jnu.12467

$\mathrm{Li}, \mathrm{X}$., Zhou, Y., \& Xu, X. (2020). Factors associated with the psychological well-being among front-line nurses exposed to COVID-2019 in China: A predictive study. Journal of Nursing Management. doi: 10.1111/JONM.13146

Maharaj, S., Lees, T., \& Lal, S. (2019). Negative mental states and their association to the cognitive function of nurses. Journal of Psychophysiology, 33(3), 207-218. https://doi.org/10.1027/0269-8803/a000223.

Mo, Y., Deng, L., Zhang, L., Lang, Q., Liao, C., Wang, N., ... \& Huang, H. (2020). Work stress among Chinese nurses to support Wuhan in fighting against COVID-19 epidemic. Journal of Nursing Management Advanced Online Publication, 28(5), 1002-1009. https://doi.org/10.1111/jonm.13014

Oyama, Y., \& Fukahori, H. (2015). A literature review of factors related to hospital nurses' health-related quality of life. Journal of Nursing Management, 23(5), 661-673. doi: 10.1111/jonm.12194

Pang, Y., Dan, H., Jung, H., Bae, N., \& Kim, O. (2020). Depressive symptoms, professional quality of life and turnover intention in Korean nurses. International Nursing Review, 67(3), 387-394. https://doi.org/10.1111/inr.12600

Sirgy, M. J. (2001). Handbook of Quality-of-Life Research: An Ethical Marketing Perspective, vol. 8 Springer Science \& Business Media. Boston: MA Kluwer Academic Publishing.

Smith, G. D., Ng, F., \& Ho Cheung Li, C. (2020). COVID-19: Emerging compassion, courage and resilience in the face of misinformation and adversity. Journal of Clinical Nursing, 29(9-10), 1425. doi: 10.1111/jocn.15231

Swami, V., Horne, G., \& Furnham, A. (2020). COVID-19-related stress and anxiety are associated with negative body image in adults from the United Kingdom. Personality and Individual Differences, 170, 110426.

Tran, T. T. T., Nguyen, N. B., Luong, M. A., Bui, T. H. A., Phan, T. D., Ngo, T. H., ... \& Nguyen, T. Q. (2019). Stress, anxiety and depression in clinical nurses in Vietnam: A cross-sectional survey and cluster analysis. International Journal of Mental Health Systems, 13(1), 3.

Wu, S., Lin, S., Li, H., Chai, W., Zhang, Q., Wu, Y., \& Zhu, W. (2014). A study on workplace violence and its effect on quality of life among medical professionals in China. Archives of Environmental \& Occupational Health, 69(2), 81-88.

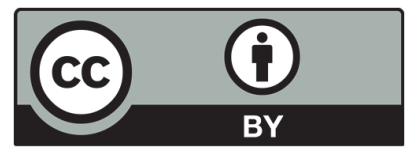

(C) 2021 by the authors; licensee Growing Science, Canada. This is an open access article distributed under the terms and conditions of the Creative Commons Attribution (CC-BY) license (http://creativecommons.org/licenses/by/4.0/). 